\title{
Anticaries effect of low-fluoride dentifrices with phosphates in children: A randomized, controlled trial
}

\author{
I.R. Freire ${ }^{a, b}$, J.P. Pessan ${ }^{a, b}$, J.G. Amaral ${ }^{a, b}$, C.C.R. Martinhon ${ }^{a, b}$, R.F. Cunha ${ }^{a, b}$, \\ A.C.B. Delbem ${ }^{\mathrm{a}, \mathrm{b}, *}$ \\ a Department of Pediatric Dentistry and Public Health, Araçatuba Dental School, Univ. Estadual Paulista (UNESP), Araçatuba, SP, Brazil \\ ${ }^{\mathrm{b}}$ Rua José Bonifácio, 1193, 16015-050 Araçatuba, SP, Brazil
}

\section{A R T I C L E I N F O}

\section{Article history:}

Received 9 December 2015

Received in revised form 26 April 2016

Accepted 28 April 2016

\section{Keywords:}

Fluoride dentifrice

Dental caries

Deciduous dentition

Polyphosphates

Sodium trimetaphosphate

Calcium glycerophosphate

\section{A B S T R A C T}

Low-fluoride dentifrices (LFD) have been recommended for young children aiming to minimize excessive fluoride intake during tooth brushing. Given the uncertainties surrounding the clinical efficacy of such formulations, alternatives to increase their anticaries effect have been investigated.

Objectives: This double-blind, randomized controlled trial assessed the clinical efficacy of LFDs supplemented with Calcium Glycerophosphate (CaGP) or Sodium Trimetaphosphate (TMP) on the progression of dental caries in the deciduous dentition.

Methods: Children (average age 48 months old) from two Brazilian cities (Araçatuba and Fernandópolis) were randomly assigned into 3 groups, according to the dentifrice to be used: 500 ppm F plus $1 \%$ TMP (“500TMP”, n= 206), 500 ppm F plus 0.25\% CaGP (“500CaGP, n= 201) and 1100 ppm F ('1100F', n=193). Clinical exams (dmfs) were performed at baseline and 18 months after dentifrices started to be used, and the increment in the number of carious lesions (final dmfs - initial dmfs) was calculated. Data were analyzed by multivariate linear regression analysis to verify the influence of city, gender, previous caries experience and type of dentifrice on dmfs increment $(\mathrm{p}<0.05)$.

Results: Mean caries increment observed for 500TMP $(0.26)$ was significantly lower when compared with $1100 \mathrm{~F}(0.74)$, while values found for 500CaGP (0.54) were not significantly different from 1100F. Caries increment was significantly higher in children from Araçatuba and in those with previous caries experience.

Conclusion: The results indicate that clinical efficacy of LFDs supplemented with TMP is superior to that observed for a conventional formulation (1100F), while the addition of CaGP leads to similar efficacy when compared to $1100 \mathrm{~F}$.

Clinical Significance: Children brushing with 500 ppm F toothpastes containing phosphate salts developed fewer caries lesions when compared with those using a $1100 \mathrm{ppm}$ F dentifrice. The tested toothpastes can be regarded as a safe alternative to conventional formulations for children under 6 years of age, based on risk-benefit considerations.

(๖ 2016 Elsevier Ltd. All rights reserved.

\section{Introduction}

The decrease in caries incidence and prevalence observed around the world has been mainly attributed to the consumption of fluoridated water and to the regular use of fluoridated dentifrices [1,2]. The widespread use of dentifrices, however, has been associated to the increase in the prevalence of dental

\footnotetext{
* Corresponding author at: Department of Pediatric Dentistry and Public Health, Araçatuba Dental School, Univ. Estadual Paulista (UNESP), Araçatuba, SP, Brazil.

E-mail addresses: adelbem@foa.unesp.br, adelbem@pq.cnpq.br (A.C.B. Delbem).
}

fluorosis, especially when products containing $1000 \mathrm{ppm} F$ or above are used by children under 5-6 years of age [3].

Alternatives for reducing the amount of fluoride ingested include the reduction of the amount of dentifrice loaded on the toothbrush, as well as the supervision of children during toothbrushing regarding the amount of toothpaste used and the need to expectorate after brushing. Given that these measures depend primarily on patient's and caregivers' compliance, the impact of such strategies on fluoride intake is uncertain, so that the use of low-fluoride dentifrices (LFDs) by children under 6 years of age has been proposed [4,5]. Nevertheless, considering the lack of scientific consensus regarding the anticaries efficacy of these formulations when compared to conventional dentifrices 
(1000-1100 ppm F), alternatives as the reduction of the $\mathrm{pH}$ [6-8] or the addition of polyphosphate salts $[9,10]$ to LFDs have been investigated.

The addition of sodium trimetaphosphate (TMP) to a LFD (500 ppm F) was shown to significantly reduce enamel mineral loss when compared to a conventional dentifrice in vitro [9,11] and in situ [12]. Similarly, the addition of calcium glycerophosphate (CaGP) to a LDF promoted similar anticaries effect to that attained after using a conventional dentifrice, according to in vitro [10] and in situ $[13,14]$ studies. Despite the promising results reported for TMP- and CaGP-containing toothpastes, no clinical evidence is still available to attest the anticaries effects of LFDs supplemented with phosphate salts on the progression of caries lesions.

Therefore, the aim of the present study was to assess the anticaries efficacy of LFDs supplemented with TMP or CaGP on the primary dentition, in a randomized, controlled trial. Based on the above-mentioned in vitro and in situ data, the study's hypothesis was that the clinical efficacy of the LFDs containing phosphates would be similar or superior to that obtained after using a conventional formulation. The American Dental Association criteria for determination of efficacy in product evaluation were adopted [15].

\section{Material and methods}

\subsection{Study design}

This randomized clinical trial was approved by the IRB of Araçatuba Dental School (Protocol 2006-014112). The study population comprised children between 18 and 60 months old, attending public kindergartens of 2 cities of the State of São Paulo, Brazil. The aims were detailed explained to parents/caregivers and signed informed consent was obtained prior to the beginning of the study. Children were randomly assigned into 3 treatment groups, according to the dentifrice to be used, following a doubleblind protocol. Parents/caregivers and children were instructed on oral hygiene procedures, brushing frequency and amount of dentifrice to be used. Caries status (dmfs) was determined at the beginning of the study and 18 months after using the dentifrices.

\subsection{Study areas}

The study was conducted in two cities of the State of São Paulo (Southeast macro region of Brazil): (1) Araçatuba, latitude $21^{\circ} 12^{\prime} 32^{\prime \prime}$ south and longitude $50^{\circ} 25^{\prime} 58^{\prime \prime}$ west, mean annual temperature $27.0^{\circ} \mathrm{C}$, Human Development Index 0.848 and mean fluoride concentration in the public water supply of $0.7 \mathrm{mg} / \mathrm{L}$; (2) Fernandópolis, latitude $20^{\circ} 17^{\prime} 02^{\prime \prime}$ south and longitude $50^{\circ} 14^{\prime} 47^{\prime \prime}$ west, mean annual temperature $25.5^{\circ} \mathrm{C}$, Human Development Index 0.823 and mean fluoride concentration in the public water supply of $0.7 \mathrm{mg} / \mathrm{L}$.

\subsection{Sample size determination and selection of volunteers}

Based on a pilot study assessing dmfs scores in 50 children from 18 to 60 months old ( mean $=0.1, S D=0.48$ ), a sample size of 125 children for each treatment group was determined $(\alpha=0.05$ e $\beta=0.2$ ), considering a dropout rate of $20 \%$ at the end of the clinical trial.

The inclusion criteria were: (1) being a resident of Araçatuba or Fernandópolis; (2) age between 18 and 60 months old; (3) parents should sign the informed consent document; (4) children should present good general health status and not taking medicines likely to interfere with salivary flow and/or biofilm formation; and (5) not having participated in another clinical trial at least 3 months prior to the beginning of the study. The exclusion criteria were: (1) voluntary withdrawal; (2) use of mouthrinses or dentifrices different to those provided by the researchers; and (3) not following up the experimental protocol (assessed by periodic visits to kindergartens). Children were selected from public kindergartens from urban areas of the 2 above-mentioned cities, totaling 747 children from 15 kindergartens which were randomly selected from a list provided by the Education Department of each city. Among those, informed consent was obtained from 600 children who fulfilled the inclusion criteria. For random allocation to the groups, classrooms were considered as units of draw (conglomerate sampling), by simple drawing using the Microsoft Excel software, so that only 1 type of dentifrice was distributed in each classroom. Allocation was done by one investigator not involved in the clinical examinations (ACBD). This planning facilitated the controlled delivery and collection of the dentifrices by the classroom's teacher, as well as daily supervised toothbrushing at school. The flowchart with the distribution of children at the beginning and at the end of the study is presented in Fig. 1.

\subsection{Dentifrice formulation}

Experimental dentifrices contained the following components: titanium dioxide, carboxymethylcellulose, methyl- $p$-hydroxybenzoate, sodium saccharin, Tutti-frutti flavoring, glycerol, hydrated silica, sodium lauryl sulfate, and water. Sodium fluoride (NaF, Merck $^{\mathbb{R}}$, Darmstadt, Germany) was added to the formulations at $500 \mu \mathrm{g} \mathrm{F} / \mathrm{g}$ (test dentifrices) and $1100 \mu \mathrm{g} \mathrm{F} / \mathrm{g}$ (positive control). Sodium trimetaphosphate (TMP, Sigma ${ }^{\mathrm{TM}}$-Aldrich Co., USA) or Calcium glycerophosphate (CaGP, Sigma ${ }^{\mathrm{TM}}$-Aldrich Co., USA, dl, $50 \% \alpha$ - and $50 \% \beta$-isomer) were added to the $500 \mu \mathrm{g} \mathrm{F} / \mathrm{g}$ formulations at concentrations of $1 \%$ and $0.25 \%$, respectively. Prior to the beginning of the study, dentifrices were submitted to laboratory tests regarding their composition, $\mathrm{pH}$, density, foaming ability, abrasiveness, consistency, rheological properties, concentration of fluoride, calcium and phosphate, as well as toxicological and microbiological tests, performed by the Brazilian Dental Association (Associação Brasileira de Odontologia - ABO) and by BITUFO Dental Products (Industry and commerce, Itupeva, SP, Brazil). All dentifrices were identical regarding their color, taste and appearance of the tube, and were coded by one author not involved in the clinical examinations (ACBD) so that the protocol was double-blind (children and examiners).

\subsection{Study protocol}

At the beginning of the study, school directors, teachers, and children's parents were instructed about the study protocol. Parents were asked to brush their children's teeth for 1 min at least twice a day at home (in the morning and before going to bed), with no restrictions regarding rinsing procedures. The amount of dentifrice to be used was standardized as the size of a rice grain ("smear"). For standardization of brushing procedures and the amount of dentifrice to be used, parents/caregivers were also instructed during a lecture.

Kits for oral hygiene contained 1 children's toothbrush, 4 dentifrice tubes (50g each) and 1 leaflet about oral hygiene care and compliance need, which were replaced every three months. Compliance was facilitated by supplying the dentifrices to all family members, in order to guarantee that children would use the experimental dentifrice. Moreover, extra kits were available at the schools if families needed them to be replaced prior to the regular quarterly supplying periods. Besides the kits for home use, toothbrushes and dentifrices were also available for use at kindergartens, for toothbrushing once/day after the main meal. 


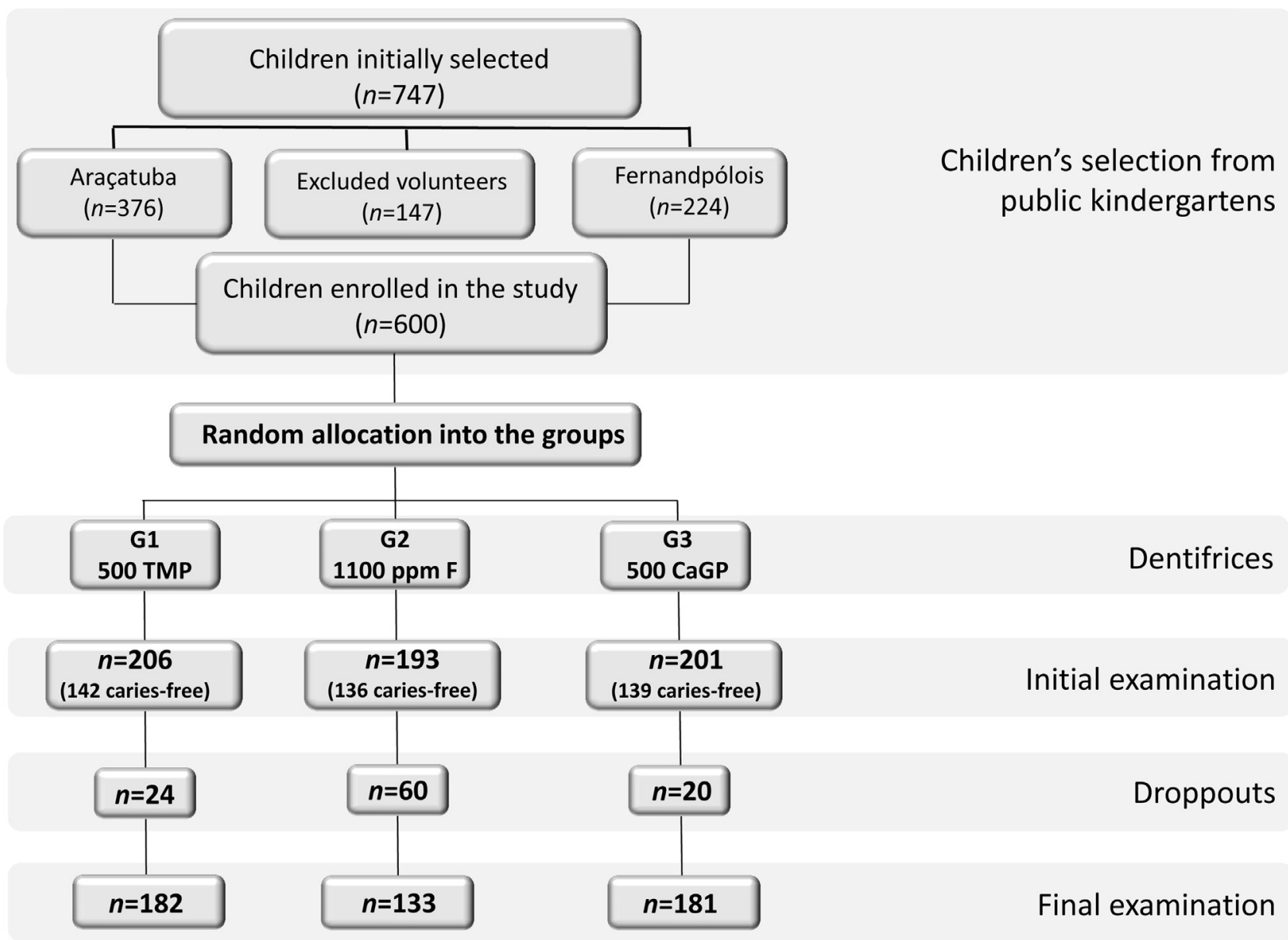

Fig. 1. Flowchart of the experimental design, showing the random allocation of the children into the groups according to the dentifrices used.

Therefore, children were expected to brush their teeth at least twice/day.

\subsection{Clinical examinations}

Children were examined at schools by 2 calibrated dentists, at the beginning of the study and every 6 months, totaling 4 examinations. The dmfs index (decayed, missed, filled surfaces) was used, following the criteria and codes established by the World Health Organization [16], by visual inspection using a plane mirror and a ballpoint probe. Tooth surfaces were cleaned by toothbrushing performed by the professional and dried with gauze, to improve the visualization of tooth surfaces. The intra- and interexaminer reliability was measured using kappa on a surface basis. The intra-examiner agreement was tested by duplication of $30 \%$ of the examinations for examiner $1(\kappa=0.97, \mathrm{p}=0.982, \mathrm{CI}=0.9694-$ $0.9828)$ and $21 \%$ of the examinations for the examiner $2(\kappa=0.95$, $\mathrm{p}=0.969, \mathrm{CI}=0.9250-0.9861)$. The inter-examiner reliability was also evaluated; twenty children were examined twice by both dentists in each survey. The related kappa values were 0.85 and 0.87 for the first and second examinations, respectively. Initial and final dmfs scores were used for the calculation of caries increment (final dmfs - initial dmfs).

\subsection{Statistical analysis}

For the statistical analysis, SPSS program version 17 (SPSS Inc., Chicago) was used, with the significance level set at $5 \%$. KruskalWallis test was used to verify differences among the groups regarding mean age at baseline. Multivariate linear regression test was applied to verify the influence of socioeconomic characteristics (city and gender), caries status at baseline and type of dentifrice upon caries increment at the end of the trial.

\section{Results}

Mean (SD) age of the children at the beginning of the study was 48 (12) months. Each group included a similar number of children of both genders and similar age range (Table 1). At baseline, children with previous caries experience corresponded to 31.1, 29.5 and $30.8 \%$ of groups who used dentifrices containing $500 \mathrm{ppm} \mathrm{F}$ plus $1 \%$ TMP (500TMP), 500 ppm F plus $0.25 \% \mathrm{CaGP}$ (500CaGP) and $1100 \mathrm{ppm} F(1100 \mathrm{~F})$, respectively. At the end of the follow-up period, the corresponding percentages reduced at a similar rate among the groups, with values of $26.4,24.8$ and $23.8 \%$, respectively; dropout rates were higher in children with previous caries experience (32\%) when compared with those from children who were caries-free at baseline (11\%). The overall dropout rate at the end of the study was $17 \%$, corresponding to 12,10 and $31 \%$ for 500TMP, 500CaGP and 1100F groups, respectively.

Mean (SD) initial dfms for children who completed the study was 1.10 (3.23) for all groups together; the corresponding values for children from Araçatuba and Fernandópolis were 1.2 (3.4) and 1.0 (3.0), respectively. Variations in initial dmfs among the groups were small, with values of 1.12 (3.31), 0.98 (4.67) and 1.17 (3.41), respectively for 500TMP, $1100 \mathrm{~F}$ and $500 \mathrm{CaGP}$, as shown in Table 1 . At the end of the 18-month follow-up period, the lowest dmfs increment was observed for 500TMP (0.26), which was $\sim 3$-fold 
Table 1

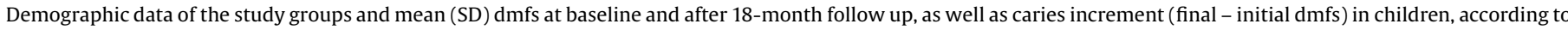
the dentifrices used.

\begin{tabular}{|c|c|c|c|c|c|c|}
\hline \multirow[t]{2}{*}{ Dentifrices } & \multirow{2}{*}{$\begin{array}{l}\text { Gender } \\
\text { (male/female) }\end{array}$} & \multicolumn{2}{|l|}{ Baseline } & \multicolumn{3}{|c|}{ 18-month follow-up } \\
\hline & & Age in months (SD) & dmfs (SD) & $\overline{n^{a}}$ & dmfs (SD) & dmfs increment (SD) \\
\hline 500 TMP & $102 / 104$ & $\begin{array}{l}48 \\
(13)\end{array}$ & $\begin{array}{l}1.12 \\
(3.31)\end{array}$ & 182 & $\begin{array}{l}1.37 \\
(3.47)\end{array}$ & $\begin{array}{l}0.26 \\
(0.73)\end{array}$ \\
\hline 500CaGP & $100 / 101$ & $\begin{array}{l}49 \\
(13)\end{array}$ & $\begin{array}{l}1.17 \\
(3.41)\end{array}$ & 181 & $\begin{array}{l}1.70 \\
(3.72)\end{array}$ & $\begin{array}{l}0.54 \\
(1.42)\end{array}$ \\
\hline 1100 ppm F & $94 / 99$ & $\begin{array}{l}50 \\
(11)\end{array}$ & $\begin{array}{l}0.98 \\
(4.67)\end{array}$ & 133 & $\begin{array}{l}1.72 \\
(6.00)\end{array}$ & $\begin{array}{l}0.74 \\
(1.90)\end{array}$ \\
\hline
\end{tabular}

500 TMP: dentifrice containing 500 ppm F (as sodium fluoride) supplemented with $1 \%$ Sodium Trimetaphosphate.

500CaGP: dentifrice containing 500 ppm F (as sodium fluoride) supplemented with 0.25\% Calcium Glycerophosphate.

1100 ppm F: dentifrice containing 1100 ppm F (as sodium fluoride), with no addition of phosphates.

* No significant differences among the groups regarding mean age at baseline (Kruskal-Wallis, $\mathrm{p}<0.05$ ).

a Indicates number of children who completed the study, excluding dropouts.

lower than that observed for $1100 \mathrm{~F}$ (0.74). Caries increment for children brushing with $500 \mathrm{CaGP}(0.54)$ was $27 \%$ lower than values found for $1100 \mathrm{~F}$, but higher than that for 500TMP.

Table 2 presents the results of the multivariate linear regression analysis, with dmfs increment as the dependent variable. City, previous caries experience at baseline and type of dentifrice significantly affected dmfs increment, while gender had no significant influence on the results. In summary, caries increment was significantly higher in children from Araçatuba and for those with previous caries experience at baseline. The variable "type of dentifrice" exerted most influence on dmfs; children who brushed with 500TMP had significantly lower caries increment when compared with $1100 \mathrm{~F}$, while no significant effect was seen for $500 \mathrm{CaGP}$ in comparison with $1100 \mathrm{~F}$. The adjusted $\mathrm{r}^{2}$ value was 0.038 .

\section{Discussion}

While the evidence of fluoridated dentifrices on the prevention of dental caries in children and adolescents is unquestionable [17], the clinical efficacy of LFDs (500-550 $\mu \mathrm{g} \mathrm{F} / \mathrm{g}$ ) is still inconclusive according to a Cochrane meta-analysis [18]. This has intensified investigations attempting to produce formulations with lower fluoride content, but with similar clinical efficacy when compared to conventional (above $1000 \mathrm{ppm} F$ ) formulations [7-9,14,19-21]. In the present study, the LFDs promoted lower caries increment when compared to the conventional formulation in children living in optimally fluoridated areas, thus confirming the study's hypothesis.

Previous clinical studies showed that a fluoride-free dentifrice containing 3\% TMP significantly reduced caries progression when compared with a dentifrice without TMP [18], but no additional effect was observed when TMP was added to fluoridated (1000$1500 \mu \mathrm{g} \mathrm{F} / \mathrm{g}$ ) toothpastes [22]. Contradictory evidence was also shown for formulations containing CaGP: while the anticaries effect of a $1000 \mu \mathrm{g} / \mathrm{g}$ toothpaste (as MFP) was significantly enhanced by the addition of $0.13 \%$ CaGP [23], an in situ study showed no additional protective effect of a $1500 \mu \mathrm{g} \mathrm{F} / \mathrm{g}$ toothpaste (MFP) when $0.13 \%$ CaGP was added to the formulation [24]. Unlike the above-mentioned formulations, the present study tested the addition of polyphosphate salts to dentifrices with low fluoride content (containing $\mathrm{NaF}$ as the fluoride source), in which the optimum molar ratios between fluoride and TMP or CaGP had been previously determined in vitro [9-11] and confirmed in situ [1214].

In brief, the anticaries effect of organic and inorganic polyphosphate salts is related to their ability in interacting with tooth enamel [25-27]. TMP is an inorganic polyphosphate able to bind to phosphate sites on enamel surface [25,27] and remain absorbed for a longer time when compared to other phosphates [25]. This interaction leads to the formation of a protective layer on the enamel surface, which limits acid diffusion and enhances calcium and fluoride diffusion into enamel $[9,11,28]$. In vitro $[9,11]$ and in situ [12] studies confirm this observation. When TMP is

Table 2

Multivariate linear regression analysis of factors associated with caries increment.

\begin{tabular}{|c|c|c|c|c|c|c|}
\hline Independent variables & $\mathrm{B}$ & Standard error of B & $\beta$ & $\mathrm{t}$ & $\mathrm{p}$ & Adjusted $r^{2}$ \\
\hline \multicolumn{6}{|c|}{ Level 1: Sociodemographic caracteristics } & 0.038 \\
\hline \multicolumn{7}{|l|}{$\begin{array}{l}0=\text { Araçatuba } \\
1=\text { Fernandópolis }\end{array}$} \\
\hline $\begin{array}{l}\text { Gender } \\
\begin{array}{l}0=\text { Female } \\
1=\text { Male }\end{array}\end{array}$ & 0.20 & 0.13 & 0.07 & 1.57 & 0.117 & \\
\hline $\begin{array}{l}\text { Level 2: Caries status a } \\
\begin{array}{l}0=\text { No } \\
1=\text { Yes }\end{array}\end{array}$ & $0.31^{\mathrm{a}}$ & 0.14 & 0.10 & 2.15 & 0.032 & \\
\hline \multicolumn{7}{|c|}{ Level 3: Type of dentifrice ( $1100 \mathrm{ppm} \mathrm{F}=0)$} \\
\hline $500 \mathrm{TMP}$ & $-0.47^{\mathrm{a}}$ & 0.16 & -0.16 & -2.98 & 0.003 & \\
\hline 500CaGP & -0.17 & 0.16 & -0.06 & -1.06 & 0.290 & \\
\hline
\end{tabular}

a Indicates significant difference between cities and among treatments. 
combined with sodium fluoride, there is a synergistic effect that depends on the proportion between these salts [9,29]. Associations between TMP: $F$ at molar ratios between $1.2: 1$ e 3.7:1 tend to produce lower mineral loss. In vitro [11] and in situ [12] data support this observation, given that the association of $500 \mathrm{ppm} F$ to 1 or $3 \%$ TMP led to higher protective effect against enamel demineralization when compared to a conventional formulation. The above-cited study also showed that fluoride and calcium concentrations in enamel and in the biofilm formed on enamel are similar to those attained with the 1100F without TMP.

CaGP is an organic polyphosphate and, as reported for TMP, also has the ability to adsorb to enamel surfaces through the phosphate radicals of its molecule $[25,26]$. CaGP, however, acts at different levels of the demineralization process [30]. When this organic phosphate adsorbs to enamel surfaces, virtually all phosphate groups are neutralized by calcium and hydroxyl groups of hydroxyapatite molecules [26], so that calcium ions from CaGP are released next to enamel surfaces. As for TMP, an adequate molar ratio between CaGP and fluoride must be determined, given that higher or lower CaGP:F ratios result in different reactions with tooth enamel in vitro [10]. Previous in vitro experiments demonstrated that the best GaGP:F ratio was 2.2:1 (CaGP at $0.25 \%$ when added to a $500 \mathrm{ppm} F$ formulation) [10], which was confirmed by in situ trials $[13,14]$. These studies showed that besides the anticaries effect produced by the interaction of CaGP with tooth enamel (in vitro), the amounts of calcium and phosphate in the biofilm formed in the presence of the 500CaGP toothpaste were similar to those obtained by $1100 \mathrm{~F}$ (in situ). Moreover, fluoride in enamel and in the biofilm formed in the presence of 500CaGP were similar to those resulting from the use of $1100 \mathrm{~F}$, indicating that fluoride was available in the oral environment during toothbrushing with the CaGP-containing toothpaste. This is paramount considering concerns regarding fluoride solubility in the product when a calcium source is added [5].

Caries increment was also shown to be influenced by city and caries experience at baseline. As for the city, this aspect was somehow unexpected considering the similarities regarding latitude, mean annual temperature, access to fluoridated drinking water, and human development index between the two locations. Moreover, and most importantly, mean baseline dmfs was very similar between the two cities, what suggests that other variables not assessed in the study might have influenced on the results. Another unexpected finding was the unusual high dropout rate seen for children using the conventional (1100F) toothpaste, considering that all formulations were identical regarding taste, color and consistency. Given that no complaints were reported by parents/caregivers throughout the study, it is likely that such findings occurred by chance. Regarding caries experience at baseline, our data is in line with previous observations for the permanent dentition that the effect of fluoride toothpaste increases with higher baseline levels of DMFS [17]. Taken together, these data reinforce the concept that for part of the population (caries-free children), the use of LFDs may be a viable option, given that it considers risks and benefits for both caries control and fluoride intake during toothbrushing $[3,5,18]$.

According to the ADA, a dentifrice is considered as superior to another when ( 1$)$ there is a significant difference $(p<0.05)$ between the test and control products; and (2) if this difference is not higher than $90 \%$ of the mean value obtained for the control dentifrice [15]. In the present study, 500TMP dentifrice met both criteria, therefore being classified as superior to the positive control (1100F). For the 500CaGP dentifrice, however, only the second criterion was met, so that the 500CaGP can be considered as equivalent to the control. Such equivalence is extremely relevant from a clinical point of view, based on risk-benefits considerations.
Concerns about excessive fluoride intake, particularly by children under 3 years of age, have prompted investigators to search for alternatives for the conventional formulations available. Besides achieving similar or superior anticaries effects when compared to the conventional dentifrice, both LFDs tested in the present study were also able to significantly reduce fluoride intake from the dentifrice during toothbrushing, as verified in a parallel study conducted with a subgroup of the children who were enrolled in the present research [31]. The systemic effect of this reduction was also observed in the resulting fluoride levels in finger- and toenails of the children [31], which are good indicators of fluoride absorption [32-34]. In this sense, it should be noted that the addition of polyphosphates is only one of the possibilities to increase the anticaries effect of LFDs. Promising results have been reported for LFDs at acidic $\mathrm{pH}$, which have been shown to increase the reactivity with tooth enamel [20], besides increasing fluoride concentrations in enamel [35], biofilm [32] and in the biofilm fluid [36] in comparison with a neutral $1100 \mathrm{ppm} F$ formulation. Acidic LFDs have been tested in two randomized controlled clinical trials, which was shown to have a similar effect on caries progression when compared to $1100 \mathrm{~F}$ dentifrices [8-37], at the same time as it significantly reduced fluoride intake and its subsequent absorption and accumulation in fingernails [32]. Besides the above-mentioned possibilities, other strategies should be encouraged so that professionals can be more confident when recommending LFDs to children at the age risk for dental fluorosis.

In summary, the present study provides robust data on the efficacy of LFDs in comparison with a conventional dentifrice, therefore being possible to accept the study's hypothesis. To attest the superiority or equivalence of new toothpastes according to the ADA guidelines, however, at least two independent clinical studies are needed. Thus, future studies are still necessary, ideally using diagnostic criteria that take into account clinical signs of dental caries that precede cavitation, aiming at assessing the effects of the products not only in caries progression, but also in the reversal of non-cavitated lesions.

\section{Acknowledgements}

This study was supported by FAPESP (São Paulo Research Foundation, Brazil), Grant \# 2010/17546-8. The corresponding author holds a patent request for a product used in the study, by the National Institute of Industrial Property - INPI/SP, on 04/29/2008 under number 018080026091, PI0801811-1, and published on January 11, 2011.

\section{References}

[1] D. Browne, H. Whelton, D. O'Mullane, Fluoride metabolism and fluorosis, J. Dent. 33 (2005) 177-186.

[2] J. Clarkson, R.G. Watt, A.J. Rugg-Gunn et al., Proceedings: 9th World Congress on Preventive Dentistry (WCPD): Community Participation and Global Alliances for Lifelong Oral Health for All, Phuket, Thailand, September 7-10, 2009, Adv. Dent. Res. 22 (2009) 2-30.

[3] M.C. Wong, A.M. Glenny, B.W. Tsang, E.C. Lo, H.V. Worthington, V.C. Marinho, Topical fluoride as a cause of dental fluorosis in children, Cochrane Database Syst. Rev. (2010) CD007693.

[4] F.V. Vilhena, H.M. Silva, S.H. Peres, M.L. Caldana, M.A. Buzalaf, The drop technique: a method to control the amount of fluoride dentifrice used by young children, Oral Health Prev. Dent. 6 (2008) 61-65.

[5] J.P. Pessan, K.J. Toumba, M.A. Buzalaf, Topical use of fluorides for caries control, Monogr. Oral Sci. 22 (2011) 115-132.

[6] F.L. Brighenti, A.C. Delbem, M.A. Buzalaf, F.A. Oliveira, D.B. Ribeiro, K.T. Sassaki, In vitro evaluation of acidified toothpastes with low fluoride content, Caries Res. 40 (2006) 239-244.

[7] M. Nobre dos Santos, L.K. Rodrigues, A.A. Del Bel Cury, J.A. Cury, In situ effect of a dentifrice with low fluoride concentration and low $\mathrm{pH}$ on enamel remineralization and fluoride uptake, J. Oral Sci. 40 (2007) 147-154.

[8] F.V. Vilhena, K.P. Olympio, J.R. Lauris, A.C. Delbem, M.A. Buzalaf, Low-fluoride acidic dentifrice: a randomized clinical trial in a fluoridated area, Caries Res. 44 (2010) 478-484. 
[9] E.M. Takeshita, L.P. Castro, K.T. Sassaki, A.C. Delbem, In vitro evaluation of dentifrice with low fluoride content supplemented with trimetaphosphate, Caries Res. 43 (2009) 50-56.

[10] A.C. Zaze, A.P. Dias, K.T. Sassaki, A.C. Delbem, The effects of low-fluoride toothpaste supplemented with calcium glycerophosphate on enamel demineralization, Clin. Oral Invest. 18 (2014) 1619-1624.

[11] E.M. Takeshita, R.A. Exterkate, A.C. Delbem, J.M. ten Cate, Evaluation of different fluoride concentrations supplemented with trimetaphosphate on enamel de- and remineralization in vitro, Caries Res. 45 (2011) 494-497.

[12] E.M. Takeshita, M. Danelon, L.P. Castro, K.T. Sassaki, A.C. Delbem, Effectiveness of a toothpaste with low fluoride content combined with trimetaphosphate on dental biofilm and enamel demineralization in situ, Caries Res. 49 (2015) $394-$ 400.

[13] A.C. Zaze, A.P. Dias, J.G. Amaral, M.L. Miyasaki, K.T. Sassaki, A.C. Delbem, In situ evaluation of low-fluoride toothpastes associated to calcium glycerophosphate on enamel remineralization, J. Dent. 42 (2014) 1621-1625.

[14] J.G. Amaral, K.T. Sassaki, C.C. Martinhon, A.C. Delbem, Effect of low-fluoride dentifrices supplemented with calcium glycerophosphate on enamel demineralization in situ, Am. J. Dent. 26 (2013) 75-80.

[15] American Dental Association, Acceptance Program Guidelines: Determination of Efficacy in Product Evaluation, American Dental Association, 2011. http:// www.ada.org/sections/scienceAndResearch/pdfs/guide_efficacypdf.

[16] World Health Organization, Oral Health Surveys-Basic Methods, fourth edition, World Health Organization, Geneva, 1997.

[17] C. Marinho, J.P. Higgins, A. Sheiham, S. Logan, Fluoride toothpastes for preventing dental caries in children and adolescents, Cochrane Database Syst. Rev. 1 (2003) CD002278.

[18] T. Walsh, H.V. Worthington, A. Glenny, P. Appelbe, V.C. Marinho, S. Xin, Fluoride toothpastes of different concentrations for preventing dental caries in children and adolescents, Cochrane Database Syst. Rev. 7 (2010) CD007868.

[19] P. Stadtler, K. Muller-Bruckschwaiger, F. Schafer, E. Huntington, The effect of sodium trimetaphosphate on caries: a 3-year clinical toothpaste trial, Caries Res. 30 (1996) 418-422.

[20] K.M. Alves, J.P. Pessan, F.L. Brighenti, K.S. Franco, F.A. Oliveira, M.A. Buzalaf, et al., In vitro evaluation of the effectiveness of acidic fluoride dentifrices, Caries Res. 41 (2007) 263-267.

[21] D.M. Camara, M.L. Miyasaki, M. Danelon, K.T. Sassaki, A.C. Delbem, Effect of low-fluoride toothpastes combined with hexametaphosphate on in vitro enamel demineralization, J. Dent. 42 (2014) 256-262.

[22] D.M. O’Mullane, D. Kavanagh, R.P. Ellwood, R.K. Chesters, F. Schafer, E. Huntington, et al., A three-year clinical trial of a combination of trimetaphosphate and sodium fluoride in silica toothpaste, J. Dent. Res. 76 (1997) 1776-1781.

[23] P.J. Mainwaring, M.N. Naylor, A four-year clinical study to determine the caries inhibiting effect of calcium glycerophosphate and sodium fluoride in calcium carbonate base dentifrices containing sodium monofluorophosphate, Caries Res. 17 (1983) 267-276.

[24] L.M. Tenuta, M.S. Cenci, A.A. Del Bel Cury, T. Pereira-Cenci, C.P. Tabchoury, G.P. Moi, et al., Effect of a calcium glycerophosphate fluoride dentifrice formulation on enamel demineralization in situ, Am. J. Dent. 22 (2009) 278-282.

[25] C. McGaughey, E.C. Stowell, Effects of polyphosphates on the solubility and mineralization of HA: relevance to a rationale for anticaries activity, J. Dent. Res. 56 (1997) 579-587.

[26] M. Anbar, E.P. Farley, D.D. Denson, K.R. Maloney, Localized fluoride release from fluorine-carrying polyphosphonates, J. Dent. Res. 58 (1979) 1134-1145.

[27] M.E. Barbour, R.P. Shellis, D.M. Parker, G.C. Allen, M. Addy, An investigation of some food-approved polymers as agents to inhibit hydroxyapatite dissolution, Eur. J. Oral Sci. 113 (2005) 457-461.

[28] J.W. van Dijk, J.M. Borggreven, F.C. Driessens, The effect of some phosphates and a phosphonate on the electrochemical properties of bovine enamel, Arch. Oral Biol. 25 (1980) 591-595.

[29] M.M. Manarelli, A.C. Delbem, T.M. Lima, F.C. Castilho, J.P. Pessan, In vitro remineralizing effect of fluoride varnishes containing sodium trimetaphosphate, Caries Res. 48 (2014) 299-305.

[30] R.J. Lynch, Calcium glycerophosphate and caries: a review of the literature, Int Dent. J. 54 (2004) 310-314.

[31] J.G. Amaral, I.R. Freire, E.F. Valle Neto, R.F. Cunha, C.C. Martinhon, A.C. Delbem Longitudinal evaluation of fluoride levels in nails of $18-30$-month-old children that were using toothpastes with 500 and $1100 \mu \mathrm{g}$ F/g, Commun. Dent. Oral Epidemiol. 42 (2014) 412-419.

[32] M.A. Buzalaf, F.V. Vilhena, F.G. Iano, L. Grizzo, J.P. Pessan, F.C. Sampaio, The effect of different fluoride concentrations an $\mathrm{pH}$ of dentifrices on plaque and nail fluoride levels in young children, Caries Res. 43 (2009) 142-146.

[33] M.A. Buzalaf, M.H. Rodrigues, J.P. Pessan, A.L. Leite, A. Arana, R.S. Villena, F.D. Forte, F.C. Sampaio, Biomarkers of fluoride in children exposed to different sources of systemic fluoride, J. Dent. Res. 90 (2011) 215-219.

[34] M.A. Buzalaf, C.S. Massaro, M.H. Rodrigues, R. Fukushima, J.P. Pessan, G.M. Whitford, F.C. Sampaio, Validation of fingernail fluoride concentration as a predictor of risk for dental fluorosis, Caries Res. 46 (2012) 394-400.

[35] H.M. Negri, J.A. Cury, Dose-response effect of a dentifrice formulation with low fl uoride concentration: an in vitro study, Pesqui. Odontol. Bras. 16 (2002) 361365.

[36] K.Y. Kondo, M.A. Buzalaf, M.M. Manarelli, A.C. Delbem, J.P. Pessan, Effects of pH and fluoride concentration of dentifrices on fluoride levels in saliva, biofilm, and biofilm fluid in vivo Clin Oral Investig [Epub ahead of print] (2015).

[37] C.A. Cardoso, D.F. Mangueira, K.P. Olympio, A.C. Magalhães, D. Rios, H.M. Honório, F.V. Vilhena, F.C. Sampaio, M.A. Buzalaf, The effect of $\mathrm{pH}$ and fluoride concentration of liquid dentifrices on caries progression, Clin. Oral Invest. 18 (2014) 761-767. 